\title{
Discusión de dilemas morales y desarrollo progresivo del juicio moral
}

\section{Isabel Carrillo}

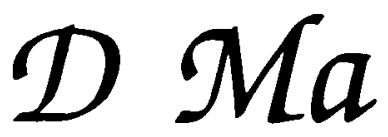

La técnica de discusión de dilemas se presenta en el marco de las teorías cognitivo-evolutivas, haciendo referencia a la teoría de los estadios morales que establece Koblberg. Se presenta un dilema dirigido en especial a los alumnos del tercer nivel de la Educación Primaria, ofreciendo, asimismo, prototipos de actividades para otros tramos educativos.

\section{LAS TEORIAS COGNITIVO-EVOLUTIVAS SOBRE EL DESARROLLO MORAL: L. KOHLBERG}

Las teorías cognitivo-evolutivas, a diferencia de las concepciones que consideran el desarrollo moral como el proceso de internalización de las normas sociales, sostienen que tal desarrollo se basa en la adquisición de principios autónomos de justicia, fruto de interacciones sociales caracterizadas por el diálogo, la cooperación y el respeto a los derechos de los demás.

El carácter «cognitivo» de estas teorías supone reconocer que la educación moral, al igual que la intelectual, tiene sus bases en la estimulación del pensamiento activo del niño y de la niña sobre cuestiones morales. Junto a ello, el término «evolutivo» permite entender los fines de la educación moral como un movimiento a través de estadios ${ }^{1}$.

L. Kohlberg se enmarca en estos enfoques cognitivo-evolutivos. Nacido en 1927, y formado en la Universidad de Chicago, Kohlberg es uno de los muchos psicólogos americanos atraídos por la obra piagetiana en los años 50 . Su trabajo es una profundización y extensión de las aportaciones de Piaget en «El criterio moral en el niño», incorporando las posteriores investigaciones que éste realizó en el campo de las categorías lógicas. La obra de Kohlberg, vinculada directamente con la teoría de Piaget, recibe también importantes influencias de diferentes filósofos, sociólogos y psicólogos (Kant, Baldwin, Mead, Dewey...). Su contribución especial ha sido aplicar el concepto de desarrollo por estadios que Piaget elaboró para el desarrollo cognitivo, al estudio del juicio moral ${ }^{2}$. En es- 
te sentido describió una serie de etapas de desarrollo del juicio moral, cada una de las cuales proporciona un sistema de razonamiento moral más complejo y, con ello, un concepto más adecuado de lo que es justo.

\section{ESTADIOS MORALES: JUICIO Y ACCION MORAL}

Los estadios morales pueden definirse como estructuras de juicio o razonamiento moral, entendiendo el juicio moral como un proceso de reflexión orientado a dar respuesta a una situación que plantea un conflicto de valores. En el juicio moral cabe diferenciar entre contenido - respuesta que da la persona, valor por el que opta-, y forma - argumentos y razones respecto a la elección realizada.

Kohlberg diferencia seis estadios de desarrollo del juicio moral estructurándolos en tres niveles. Los niveles definen los enfoques de los problemas morales, mientras que los estadios definen los criterios por los que la persona ejercita su juicio moral, es decir, cada estadio supone una forma de pensar diferente e indica cómo se piensa - no lo que se piensa-. Los niveles y estadios que establece Kohlberg son los siguientes ${ }^{3:}$

\begin{tabular}{|l|l|}
\hline \multirow{2}{*}{$\begin{array}{l}\text { NIVEL I: } \\
\text { PRECONVENCIONAL }\end{array}$} & $\begin{array}{l}\text { ESTADIO 1: Moral heterónoma-castigo/ } \\
\text { obediencia. }\end{array}$ \\
\cline { 2 - 2 } & $\begin{array}{l}\text { ESTADIO 2: Individualismo, finalidad } \\
\text { instrumental e intercambio. }\end{array}$ \\
\hline \multirow{2}{*}{$\begin{array}{l}\text { NIVEL II: } \\
\text { CONVENCIONAL }\end{array}$} & $\begin{array}{l}\text { ESTADIO 3: Relaciones interpersonales de } \\
\text { conformidad, expectativas mutuas. }\end{array}$ \\
\cline { 2 - 2 } & ESTADIO 4: Sistema social y conciencia. \\
\hline \multirow{2}{*}{$\begin{array}{l}\text { NIVEL III: } \\
\text { POSCONVENCIONAL }\end{array}$} & $\begin{array}{l}\text { ESTADIO 5: Contrato social o utilidad } \\
\text { y derechos individuales. }\end{array}$ \\
\cline { 2 - 2 } & ESTADIO 6: Principios éticos universales. \\
\hline
\end{tabular}

El desarrollo moral no se da de forma independiente, sino que un razonamiento moral avanzado dependerá de que haya un razonamiento lógico avanzado. Así, el desarrollo lógico es una condición necesaria del desarrollo moral, aunque no suficiente ${ }^{4}$. Pero además, a los estadios de desarrollo lógico siguen los estadios de percepción social o adopción de roles. Al igual que en la lógica, el desarrollo de un estadio de percepción social es anterior al desarrollo del estadio paralelo del juicio moral. Así, del mismo modo que hay una secuencia vertical de etapas en movimiento ascendente desde el estadio moral 1 hasta el estadio moral 2 y de éste al siguiente, hay también una secuencia horizontal de etapas cuyo movimiento va desde la percepción lógica a la social y de ésta al juicio moral, siendo la última etapa en esta secuencia horizontal la conducta moral. Actuar en una forma moralmente elevada exige un estadio elevado de razonamiento moral. Una persona no puede seguir unos principios morales si no los entiende o no cree en ellos. Sin embargo, se puede razonar en términos de tales principios y no vivir de acuerdo con ellos. De esta forma, la madurez del juicio 
moral se presenta también como una condición necesaria, pero no suficiente, para la madurez de la acción moral. Las personas en estadios superiores -4, 5 y 6 - orientados por principios estables, objetivos y equilibrados, son más coherentes en sus pautas de conducta que aquellas situadas en los estadios inferiores, cuya conducta va cambiando en función de factores personales y situacionales.

\section{LA DISCUSION DE DILEMAS MORALES}

La discusión de dilemas morales es una técnica de educación moral derivada de los trabajos de Kohlberg. Los dilemas son breves narraciones de situaciones que presentan un conflicto de valor, es decir, un personaje se encuentra en una situación difícil y tiene que elegir, por lo general, entre dos alternativas óptimas y equiparables 5 .

Se parte de la constatación de que no hay progreso en el juicio moral de las personas si, previamente, no experimentan un conflicto cognitivo que rompa la seguridad de sus razonamientos. Por ello, la discusión de dilemas morales pretende crear conflicto en los alumnos/as y, junto a ello, ayudarles a restablecer el equilibrio en un nivel superior de juicio moral. El conflicto cognitivo no sólo se produce mediante la presentación de dilemas, sino que la interacción con los iguales y adultos - que pueden tener razonamientos diferentes a los propios-, así como la consideración de los problemas morales desde puntos de vista diferentes también son fuente de conflicto. Es en este sentido que Kohlberg considera que es más probable que el cambio moral ocurra cuando los debates - la discusión de dilemas morales - consiguen suscitar un conflicto cognitivo entre los participantes. El interactuar con otras personas y confrontar opiniones, perspectivas..., permite replantearse las propias posiciones e iniciar un proceso de reestructuración del modo de razonar sobre cuestiones morales.

Así, mediante la discusión de dilemas morales, la persona desarrolla su capacidad de razonar, su juicio moral, sobre situaciones que presentan un conflicto de valores. En este proceso, el individuo considera sus propios valores a propósito de temas moralmente relevantes, dilucidando entre lo que considera correcto o incorrecto ${ }^{6}$.

De lo expuesto se deduce que la metodología educativa de Kohlberg para promover el desarrollo del juicio moral se basa en proponer a los alumnos/as dilemas morales que despierten su interés, preguntándoles directamente cuál sería la mejor solución para el dilema, es decir, cada alumno/a debe pensar cuál es la mejor decisión y fundamentarla en razonamientos moral y lógicamente válidos.

A continuación presentamos un dilema, a modo de ejemplo, así como algunas orientaciones para su discusión

«GASTOS EN ARMAMENTO» (Ambito temático: Los Derechos Humanos. Curso 5. E.G.B.).

Tema/Objetivo:

Con este dilema pretendemos abordar el tema de las condiciones de vida en los países en vías de desarrollo, reflexionando sobre los conflictos de valor 
derivados del elevado gasto en armamento de algunos países cuya economía es muy precaria. Este tema se puede trabajar relacionado con el estudio de la Declaración Universal de los Derechos Humanos (arts. 1 y 25), analizando las necesidades básicas de toda persona.

\section{Actividad:}

En algunos países, la falta de recursos económicos es la causa de que sus habitantes pasen muchas necesidades. Además, las guerras en las que a veces se encuentran empeoran aún más su situación. A continuación tienes un ejemplo.

«En un pequeño país situado en el desierto de Africa, la mayoría de personas viven en condiciones muy deficientes, ya que el país no tiene casi recursos. Además, en el último año, debido a la falta de lluvias, se han acabado las reservas de agua potable $y$, por este motivo -además de otros-, han aumentado las enfermedades $\mathrm{y}$ las muertes entre los niños y niñas al no disponer ni de hospitales ni de las medicinas necesarias.»

A los problemas anteriores se añade otro, la guerra. Desde hace tres años y medio, este país lucha con un país vecino por unos territorios que quedan entre ellos. Estos territorios son muy ricos, ya que tienen muchas minas y pozos de agua.»

Este país sabe que no podrá ganar la guerra con las armas que tiene, sino que tendría que comprar nuevo armamento más sofisticado y perfeccionado. Como el país no tiene mucho dinero, si compra estas armas no podrá comprar las medicinas, ni construir los hospitales necesarios, para impedir las continuas muertes, de niños y niñas y otras personas, originadas por las enfermedades y las epidemias."

La decisión que ha de tomar este país de Africa es difícil. Los territorios por los que lucha podrían mejorar su economía, y la vida de las personas, pero también tienen otros problemas urgentes que han de resolver.»

- Cuando un país tiene recursos muy limitados ha de establecer prioridades. Decidir hacer una cosa supone dejar de hacer otras que también pueden ser necesarias. Si tenemos en cuenta esto, que debería hacer este país de Africa, comprar las armas o no? ¿Por qué?

\section{Orientaciones para la discusión del dilema:}

\section{1) Presentar el dilema}

Mediante la lectura -individual o colectiva-. La presentación del dilema también puede realizarse mediante una representación de la situación que plantea, por medio de dibujos...

\section{2) Recapitulación.}

Comprobar la comprensión del dilema planteado (terminología, conflicto y alternativas) e insistir en que se ha de contestar lo que debería hacer el protagonista y no lo que probablemente haría.

3) Reflexión individual:

Cada alumno/a reflexiona, individualmente, sobre el dilema y selecciona una alternativa. La decisión adoptada, así como las razones de la misma, se expresan por escrito.

\section{4) Discusión del dilema:}

Se puede optar por realizar un comentario general a nivel de toda la clase, exponiendo cada alumno/a sus respuestas y argumentos, o realizar un proceso de discusión en pequeños grupos, pasando, posteriormente, a la discusión generalizada de todo el grupo. En este último caso el profesor/a orientará a los grupos para que centren la discusión -que no se alargará más de diez o quince 
minutos - en torno al conflicto que plantea el dilema. Antes de iniciar la discusión con toda la clase, un representante de cada grupo resumirá lo que han discutido, los argumentos expuestos en su grupo.

5) Final de la actividad:

Para finalizar la actividad puede proponerse a los alumnos/as que reconsideren su postura inicial - valorando si han experimentado alguna modificación-, que encuentren argumentos para defender la postura contraria a là elegida, que resuman las posturas, soluciones y argumentos planteados, que expongan situaciones similares a la del dilema...

\section{Ejemplos de dilemas para otras edades:}

«LOS CROMOS» (Ambito temático: Relación entre iguales. Curso: $1 .^{\circ}$ E.G.B.).

Pablo ha llevado al colegio muchos cromos que le han regalado.

Se los ha enseñado a José, su compañero.

José le dice que si no le da cromos, no será su amigo.

- ¿Qué debería hacer Pablo? ¿Por qué?

Otras cuestiones, relacionadas con el tema de la amistad, que pueden plantearse durante la discusión del dilema son: ¿Es importante que José sea amigo de Pablo? ¿Por qué?; ¿Te has encontrado alguna vez en una situación parecida? ¿Qué pasó?; Para tener amigos, ¿se ha de hacer regalos? ¿Por qué?

«EN SU CASO» (Ambito temático: Escuela y trabajo. Curso: $8 .^{\circ}$ E.G.B.) Quien ahora habla es un chico de séptimo.

«Un día, antes de un examen, alguien escribió la respuesta de una posible pregunta en la pizarra. La profesora no lo vio y comenzó la prueba. Aquella pregunta estaba. Cuando llevábamos ya unos diez minutos haciendo el examen, la profesora se dio cuenta de que la respuesta estaba escrita en la pizarra. Entonces la borró sin hacer ningún comentario.

El día que nos devolvieron los exámenes corregidos, nos preguntó quién había copiado para bajarles un punto. Mucha gente lo reconoció, pero yo no sabía qué hacer, porque había aprobado muy justo y sin ese punto podía suspender la evaluación».

Contesta estas preguntas:

- ¿Qué debería hacer este chico y por qué?

- ¿Para qué sirven los exámenes?

Orientaciones generales para la aplicación y elaboración de dilemas morales:

Aplicación: Respecto a la discusión de dilemas morales deberemos atender a los siguientes aspectos: 
a) Creación de una atmósfera adecuada, es decir, de un entorno que estimule el desarrollo moral de los alumnos/as. Para ello hay que atender a la disposición física que ha de favorecer la expresión e intercambio de opiniones (es importante que se puedan ver entre ellos y que el educador/a se encuentre como uno más del grupo), así como aquellas actitudes de respeto y diálogo que permiten una comunicación abierta.

b) El educador/a ha de tener presente que durante la discusión de dilemas morales no ha de obligar a nadie a expresar su opinión, ya que ello puede desmotivar el diálogo (en este sentido no debe olvidar que el diálogo interno también favorece el desarrollo moral). Tampoco ha de dominar la discusión, sino que debe favorecer la interacción entre alumnos/as.

c) Aparte de dialogar sobre la pregunta central del dilema en torno a la decisión que debería tomar el protagonista, se pueden plantear otras cuestiones relacionadas con el conflicto, analizar las consecuencias de cada elección, realizar transferencias a la vida real, plantear dilemas alternativos...

Elaboración: Al redactar dilemas morales no debemos olvidar que éstos han de plantear conflictos adecuados a la edad y experiencia de los alumnos/as. Así, los dilemas han de ser motivadores y controvertidos, en el sentido de que los alumnos/as puedan dar diferentes argumentos y soluciones. No hay que olvidar que la creación del conflicto cognitivo tiene como objetivo favorecer el desarrollo del juicio moral.

Para facilitar la redacción de dilemas morales se pueden seguir una serie de pautas. A continuación presentamos un ejemplo.

«FIESTA DE CUMPLEAÑOS» (Ambito temático: Relación entre iguales. Curso: $3 .^{\circ}$ E.G.B.)

\begin{tabular}{|l|l|}
\hline $\begin{array}{l}\text { Pautas para la redacción de } \\
\text { dilemas }\end{array}$ & Dilema \\
\hline $\begin{array}{l}\text { 1) CENTRARLO EN UNA SI- } \\
\text { TUACION Y DEFINIR } \\
\text { AL/LOS PROTAGONISTA/S }\end{array}$ & $\begin{array}{l}\text { Patricia es una compañera de María. En clase tie- } \\
\text { ne fama de aburrida. Antes a María también se } \\
\text { lo parecía, pero hace quince días que se sientan } \\
\text { juntas y siempre que le pide alguna cosa, Patricia } \\
\text { la ayuda. María también ve cómo Patricia deja sus } \\
\text { deberes a todos los niños que se lo piden, aunque } \\
\text { después éstos la critiquen y no le digan nada a la } \\
\text { hora de jugar. }\end{array}$ \\
\hline $\begin{array}{l}\text { 2) PLANTEAR CUESTIO- } \\
\text { NES MORALES, EL CON- } \\
\text { FLICTO }\end{array}$ & $\begin{array}{l}\text { El próximo lunes es el cumpleaños de María y hará } \\
\text { una fiesta para sus amigos. Le gustaría mucho in- } \\
\text { vitar a Patricia y cree que a ella también le haría } \\
\text { ilusión, pero sabe que a sus amigos les cae muy } \\
\text { mal y no querrían estar con ella en la fiesta. }\end{array}$ \\
\hline $\begin{array}{l}\text { 3) PLANTEAR LAS ALTER- } \\
\text { NATIVAS; PREGUNTAR } \\
\text { QUE DEBERIA HACER EL } \\
\text { PROTAGONISTA }\end{array}$ & $\begin{array}{l}\text { ¿Crees que María ha de invitar a Patricia? SI. NO } \\
\text { ¿Por qué? }\end{array}$ \\
\hline $\begin{array}{l}\text { 4) FORMULAR OTRAS PRE- } \\
\text { GUNTAS RELACIONADAS }\end{array}$ & $\begin{array}{l}\text { Busca dos razones para que María invite a Patri- } \\
\text { cia. Ahora busca dos razones más para que María } \\
\text { no invite a Patricia. }\end{array}$ \\
\hline
\end{tabular}




\section{Notas}

${ }^{1}$ Cada estadio forma un todo estructurado que implica diferencias cualitativas en el modo de pensar, es decir, un cambio de estadio supone una reestructuración de cómo uno piensa sobre toda una serie de temas morales. Además, los estadios son integraciones jerárquicas en el sentido de que, cuando una persona se desarrolla de una etapa a la siguiente, el nivel más alto reintegra las estructuras que se encuentran en niveles más bajos. En este proceso, los estadios se presentan en una secuencia invariante de forma que el pensamiento se desarrolla en dirección ascendente, hacia un mayor equilibrio.

${ }^{2}$ Kolhberg considera que el desarrollo del juicio moral se articula en una secuencia de estadios de acuerdo con los criterios de Piaget, es decir, los estadios son invariantes en el orden en que se alcanzan, son universales, forman estructuras de conjunto y están jerárquicamente interrelacionados.

${ }^{3}$ Para mayor información se pueden consultar las siguientes fuentes bibliográficas:

HERSH, R.; REIMER, J.; PAOLITTO, D. (1988). El crecimiento moral. De Piaget a Koblberg. Madrid. Narcea. Págs. 53-70.

KOLHBERG, L. «Estadios morales y moralización. El enfoque congnitivo-evolutivo» en TURIEL, E.; ENESCO, I.; LINAZA, J. (comp.) (1989). El mundo social en la mente infantil. Madrid. Alianza. Págs. 71-100.

PUIG, J.M." \& MARTINEZ, M., (1989). Educación moral y democracia. Barcelona. Laertes. Págs. 88-105.

${ }^{4}$ Algunas personas están en un estadio lógico más alto que el estadio moral paralelo, pero en esencia no hay ninguno que esté en un estadio moral más alto que su estadio lógico.

${ }^{5}$ Según su contenido, o la situación analizada, los dilemas pueden ser: A) Hipotéticos: Plantean problemas abstractos con situaciones y personajes poco definidos. Tienen poca o nula relación con el contexto real del alumno/a. B) Reales: Se extraen de acontecimientos históricos o de aquellos originados en el contex to diario de los alumnos/as.

${ }^{6} \mathrm{La}$ construcción progresiva de formas de razonamiento moral cada vez mejores atraviesa las siguientes fases: en un primer nivel, el preconvencional, la persona resuelve los conflictos morales desde la perspectiva de los intereses de los individuos implicados; en el nivel convencional los resuelve desde la perspectiva de los intereses de la sociedad; y en el posconvencional, resuelve los conflictos desde una perspectiva superior a la sociedad, es decir, desde los criterios de justicia que impone la propia conciencia.

\section{Referencias}

Berkowitz, M. W. «El papel de la discusión en educación moral». Seminario de Educación Mo. ral. Departamento de Teoría e Historia de la Educación. Facultad de Pedagogía. U. B. 9-13 de diciembre de 1991.

Hersh, R.; Reimer, J., Y Paolitto, D. (1988). El crecimiento moral. De Piaget a Koblberg. Madrid; Narcea.

KohlBerG, L. «El enfoque cognitivo-evolutivo de la educación moral», en Jordan, J. A. Y SANToLARIA, F. F. (eds.) (1987). La educación moral, boy. Cuestiones y perspectivas. Barcelona. PPU. Págs. 85-114.

- «Estadios morales y moralización. El enfoque cognitivo-evolutivo», en Turiel, E.; Enesco, I.; Linaza, J. (1989). El mundo social en la mente infantil. Madrid: Alianza. Págs. 71-100.

Marchesi, A. «El desarrollo moral», en Palacios, J.; Marchesi, A.; Carretero, M. (comp.) (1991). Psicología evolutiva 2. Desarrollo cognitivo y social del niño. Madrid: Alianza. Págs. 351-387.

Paya, M. «Discusión de dilemas», en Martínez, M. y Puig, J. M. a (coord.) (1991). La educación moral. Perspectivas de futuro y técnicas de trabajo. Barcelona: Graó. ICE. U.B. Págs. 45-47.

Puig, J. M. a Y MarTínez, M. (1989). Educación moral y democracia. Barcelona: Laertes. 
Discusión de dilemas morales y desarrollo progresivo del juicio moral Isabel Carrillo Flores CL\&E, 1992, 15, pp. 55-62

Resumen: Los estudios de L. Kohlberg se estructuran en torno a cómo la discusión de dilemas morales favorece el desarrollo del juicio moral. Los dilemas son breves narraciones de situaciones que presentan un conflicto de valor, es decir, un personaje se encuentra en una situación difícil y tiene que elegir, por lo general, entre dos alternativas óptimas y equiparables.'

Se parte de la constatación de que no hay progreso en el juicio moral de las personas si, previamente, no experimentan un conflicto cognitivo que rompa la seguridad de sus razonamientos. Por ello, la discusión de dilemas morales pretende crear conflicto en los alumnos/as y, junto a ello, ayudarles a restablecer el equilibrio en un nivel superior de juicio moral -desarrollo a través de estadios.

Datos sobre el autor: Isabel Carrillo Flores es pedagoga y actualmente goza de una beca de Formación de Personal Investigador de la Generalitat de Catalunya. También es miembro del Grupo de Investigación en Educación Moral (G.R.E.M.).

Dirección: Departamento de Teoría e Historia de la Educación, Facultad de Pedagogía, Universidad de Barcelona, Baldiri Reixac, s/n 08028 Barcelona. Tel (93) 3333466.

(C) De todos los artículos deberá solicitarse por escrito autorización de CL\&E y de los autores para el uso en forma facsímil, fotocopia o cualquier otro medio de reproducción impresa. CL\&E se reserva el derecho de interponer las acciones legales necesarias en aquellos casos en que se contravenga la ley de derechos de autor.

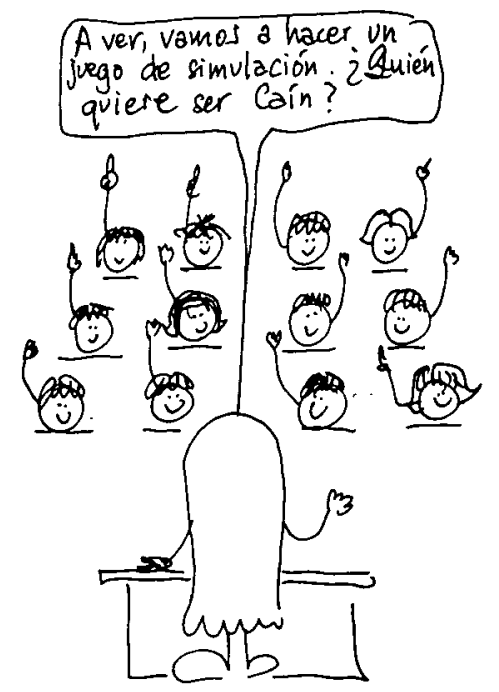

